\title{
Calibración y verificación de fórmulas para calcular el caudal en compuertas radiales con descarga sumergida
}

\section{Calibration and verification of formulas to calculate the flow in radial gates for submerged discharge}

\author{
Jiménez-Castañeda Amado Abel \\ Universidad Nacional Autónoma de México \\ Instituto de Ingeniería \\ Correo: ajc@pumas.ii.unam.mx \\ https://orcid.org/0000-0003-2081-5429 \\ Berezowsky-Verduzco Moisés \\ Universidad Nacional Autónoma de México \\ Instituto de Ingeniería \\ Correo: mbv@pumas.ii.unam.mx \\ https://orcid.org/0000-0002-7675-3450
}

\author{
Hernández-López Emmanuel \\ Universidad Nacional Autónoma de México \\ Instituto de Ingeniería \\ Correo: unam.ehl@gmail.com \\ https://orcid.org/0000-0001-9251-0785 \\ Caballero-Coranguez Christian Alberto \\ Universidad Nacional Autónoma de México \\ Instituto de Ingeniería \\ Correo: ccaballeroc@iingen.unam.mx \\ https://orcid.org/0000-0001-9080-7083
}

\section{Resumen}

Se describen los estudios desarrollados en un modelo físico para obtener la capacidad de descarga de un conjunto de compuertas radiales con desfogue sumergido. Los resultados experimentales se emplearon para calibrar y verificar un modelo numérico, el cual es útil para calcular el caudal que descarga una compuerta radial; la expresión matemática se dedujo con base en el análisis dimensional y la teoría de similitud incompleta, y es aplicable para condiciones de descarga libre o sumergida de compuertas radiales. Primero se describe el modelo físico de una estructura de control integrada por tres compuertas radiales y dos pilas; aguas abajo de las compuertas se tienen tres conductos paralelos de sección transversal rectangular, que funcionan como alcantarillas; después, se presenta el procedimiento para obtener la ley de descarga de las alcantarillas, para el caso en el que las compuertas radiales estén totalmente abiertas. Esta ley de descarga se calibra con base en mediciones experimentales, posteriormente se explica el cálculo de las curvas de descarga de las compuertas radiales con descarga sumergida y se discuten dos modelos numéricos recientes que son aplicables para condiciones de descarga libre o ahogada. El modelo numérico que mejor corresponde con los resultados experimentales es calibrado y verificado.

Descriptores: Compuertas radiales, descarga sumergida, alcantarillas.

\begin{abstract}
The studies developed in a physical model to obtain the flow rate through submerged radial gates are described. The experimental results are used to calibrate and verify a numerical model for computing the water discharge; the numerical model is based on dimensional analysis and the theory of incomplete similarity; the numerical model is useful for calculating the laws of discharge of radial gates, for both free and submerged discharge. First, the physical model of a control structure is described; there are two piles and three radial gates; downstream from the radial gates there are three parallel culverts, with rectangular cross section; after, the process to obtain the law of discharge of the culverts is explained, for the case in which the radial gates are fully open; this law of discharge is adjusted using the experimental measurements. Subsequently, the calculation of the curves of the radial gates with submerged discharge is explain; two recent numerical models that are applicable for free or submerged discharge conditions are presented. The numerical model that best match the experimental results is calibrated and verified.
\end{abstract}

Keywords: Radial gates, submerged discharge, culverts. 


\section{INTRODUCCIÓN}

Es común que en sistemas hidráulicos para drenaje urbano, distritos de riego, obras de toma para plantas potabilizadoras o de tratamiento de aguas residuales, obras hidráulicas para almacenamiento y control de avenidas, entre otros, se utilicen las compuertas radiales. Además de su uso para controlar los niveles de la superficie libre del agua en los citados sistemas, llegan a ser empleadas como estructuras de aforo; esta alternativa es útil y práctica para mejorar la administración del agua.

Actualmente, se dispone de varios métodos para el cálculo del caudal que descarga una compuerta radial; sin embargo, Henderson (1966) aclara que el modelo numérico que presenta, permite obtener resultados preliminares; Sotelo (2002) discute la incertidumbre y el desacuerdo que hay en el cálculo de los coeficientes de velocidad, de contracción y de descarga que se emplean para el cálculo del caudal que descarga una compuerta. En Jiménez y Berezowsky (2009) y Jiménez (2017) se demuestra la importancia de calibrar y verificar metodologías como la de Cozzo (1978) y Bijankhan et al. (2013), para obtener la ley de descarga de compuertas radiales, en condiciones de descarga libre; esto requiere disponer de un modelo hidráulico a escala, o bien, tener mediciones de campo, lo cual es mucho más costoso. En el caso de que la compuerta funcione hidráulicamente con descarga ahogada, se tiene mayor incertidumbre en la estimación del caudal, debido a la complejidad que presenta el comportamiento del flujo en la zona inmediatamente aguas abajo de la compuerta; los errores obtenidos con los métodos tradicionalmente empleados, pueden llegar a ser hasta del orden de $50 \%$, e incluso superiores, (Clemmens et al., 2003).

Los modelos numéricos para calcular el caudal que descarga una compuerta radial, fueron deducidos con base en uno de tres enfoques generales: el primero de ellos consiste en emplear la ecuación de la energía; otro se basa tanto en la ecuación de la energía como en la del momentum; un tercer enfoque, relativamente nuevo, consiste en aplicar el análisis dimensional junto con la teoría de la similitud incompleta, es el que emplean Bijankhan et al. (2013), quienes además de presentar el estado del arte respecto al cálculo del caudal que descarga una compuerta radial, proponen nuevos métodos que son aplicables tanto en condiciones de descarga libre como sumergida. Abdelhaleem (2016) también emplea el mismo enfoque, y propone un modelo numérico que es calibrado y verificado con base en múltiples mediciones en canales para riego con estructuras de control, que tienen instaladas compuertas radiales en paralelo, y que funcionan hidráulicamente con descarga ahogada.

\section{OBjetIVO DEL ESTUDIO}

Presentar una alternativa de cálculo para estimar la capacidad de descarga de compuertas radiales, con desfogue sumergido, así como su aplicación y el proceso de calibración y verificación de las correspondientes fórmulas adimensionales, con base en un estudio experimental.

\section{MATERIALES Y MÉTODOS DISPONIBLES}

Para este estudio, se dispone del modelo físico de una estructura de control (llamada Túnel - Alcantarilla, $T-A$ ), constituida por tres compuertas radiales colocadas en paralelo, cuya descarga es sumergida. Aguas abajo de cada compuerta se tiene una alcantarilla de sección rectangular. El empleo del modelo físico de esta estructura y de los novedosos métodos publicados por Bijankhan et al. (2013) y Abdelhaleem (2016), para calcular las leyes de descarga de las compuertas, con desfogue sumergido, permite tener los elementos necesarios para calibrar y verificar el modelo numérico más adecuado.

El cálculo de la ley de descarga de compuertas radiales con desfogue sumergido, requiere del conocimiento de la capacidad de conducción que se tiene aguas abajo de las compuertas; por ello, primero se describe la obtención de la capacidad de conducción del sistema hidráulico en estudio, para el caso en el que las compuertas estén totalmente abiertas; la ley de descarga es calibrada y verificada con base en mediciones en el modelo físico. Estos resultados permiten definir la condición de frontera cuando las compuertas están parcialmente abiertas, y la descarga es sumergida; después se desarrolla el estudio experimental con las compuertas parcialmente abiertas y descarga ahogada; posteriormente, de acuerdo con los correspondientes resultados experimentales se elabora el proceso de calibración y verificación para obtener las leyes de descarga de las compuertas radiales con desfogue sumergido.

\section{DESCRIPCIÓN GENERAL DEL MODELO Fí́ICO}

En el modelo físico de un sistema de canales se tienen incluidas varias estructuras de control; en una de ellas (el $T-A)$, hay tres compuertas radiales y dos pilas; la estructura de control sirve para derivar agua de un canal a otro. En la fotografía de la Figura 1 se observa el entorno de la estructura de control (enmarcada con color rojo). 
Las alcantarillas sirven para cruzar por debajo a un río (El Salto). En particular, la estructura de control se localiza aguas abajo de la confluencia de dos canales (Figura 1); uno de ellos corresponde al portal de salida del Túnel Emisor Central (TEC), mientras que el otro recibe el caudal que sale del Túnel Emisor Oriente (TEO, inaugurado en diciembre de 2019).

Las pilas con compuertas y la entrada de las alcantarillas se presentan del lado izquierdo de la Figura 2, y en el lado derecho de la misma figura está la salida de las tres alcantarillas.

En las Figuras 1, 2 y 3, se incluyen los marcos de los soportes y los limnímetros (indicados con las letras $A, B$ y $C)$, que fueron empleados para medir la elevación de la superficie libre del agua durante las pruebas experimentales. Además, en la Figura 3 se presenta el tramo del canal al que descarga la estructura $T-A$.

En la Figura 3 también se observa el tramo del canal que está aguas abajo de la curva horizontal; al final de este tramo se colocó una compuerta vertical plana para controlar la elevación del nivel del agua en la frontera de aguas abajo (soporte con limnímetro $C$ ).

\section{DESCRIPCIÓN DE LA ESTRUCTURA DE CONTROL Y LAS ALCANTARILLAS}

En la Figura 4a se presentan las dimensiones del modelo físico de la estructura de control, integrada por dos pilas, tres compuertas radiales y alcantarillas de sección rectangular. Las longitudes y elevaciones que se reportan se obtuvieron al hacer el levantamiento topográfico del modelo físico, escala 1:30, con una estación total. Se escoge como elevación de referencia la cota $70.0667 \mathrm{~m}$, asociada a la elevación de la plantilla del canal horizontal que se localiza en la entrada de las alcantarillas.

En la Figura $4 \mathrm{~b}$ se muestran los tres sitios $(A, B$ y $C)$ en los que se instalaron sendos limnímetros para registrar los correspondientes tirantes, en cada una de las pruebas experimentales realizadas.

El tramo del canal localizado aguas arriba de la entrada a las alcantarillas (ver fotografía del lado izquierdo en la Figura 2), es de sección transversal rectangular, con ancho de plantilla de $0.333 \mathrm{~m}$; en este canal hay dos pilas con $0.0167 \mathrm{~m}$ de ancho para sostener las compuertas; la nariz de las pilas está a $0.385 \mathrm{~m}$ de la entrada a las alcantarillas.

Cada una de las compuertas radiales tiene un ancho de $0.10 \mathrm{~m}$, con radio de $0.12 \mathrm{~m}$; la altura del perno respecto a la plantilla del canal es de $0.105 \mathrm{~m}$; hay una distancia de $0.22 \mathrm{~m}$ entre el eje del perno de las compuertas y la entrada de las alcantarillas. Ambas pilas continúan a lo largo de las alcantarillas, y exceden la longitud de cada conducto con $0.055 \mathrm{~m}$, aguas abajo. Las dos pilas forman dentro del túnel tres conductos de sección rectangular, con ancho de $0.10 \mathrm{~m}$ cada uno y altura de
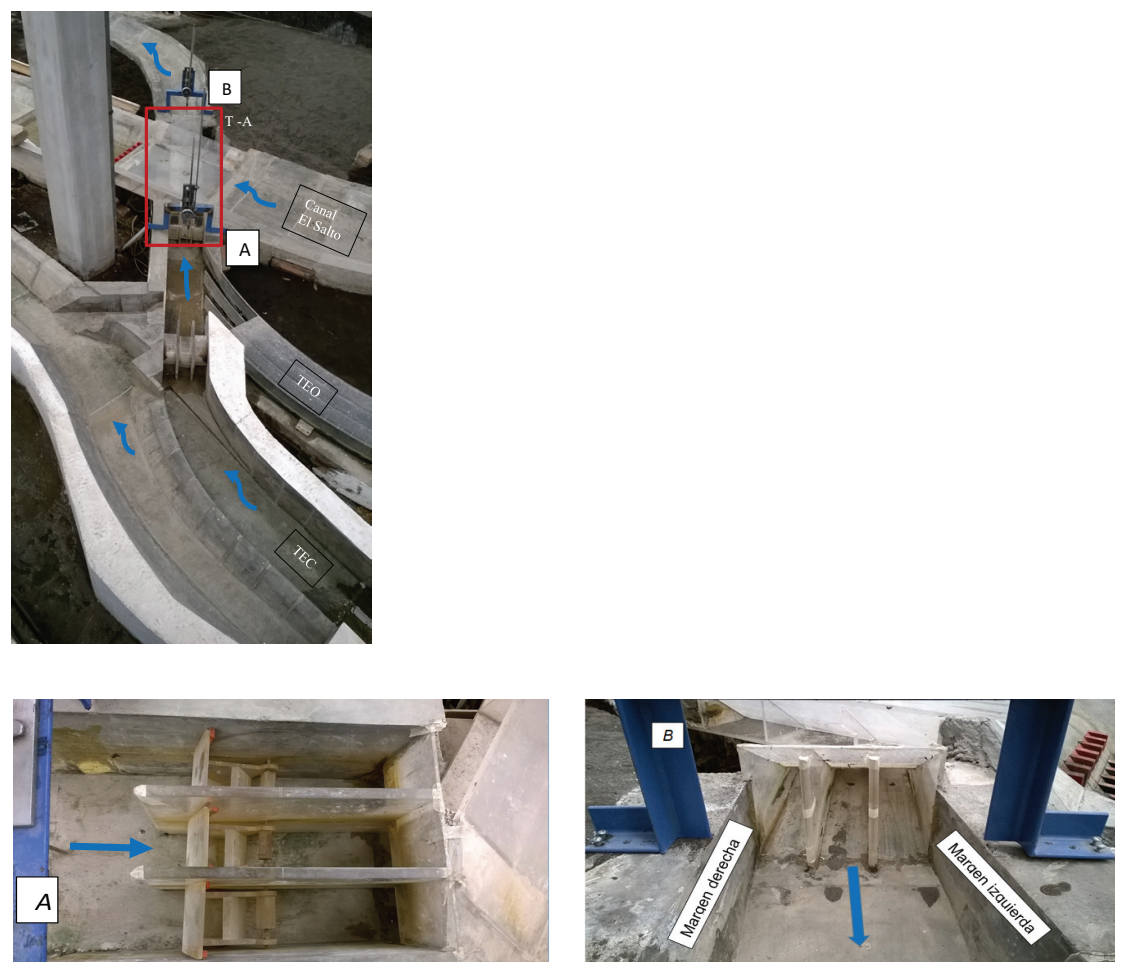

Figura 1. Entorno de la estructura de control, con pilas, compuertas y alcantarillas

Figura 2. Entrada y salida de la estructura de control y alcantarillas, respectivamente 

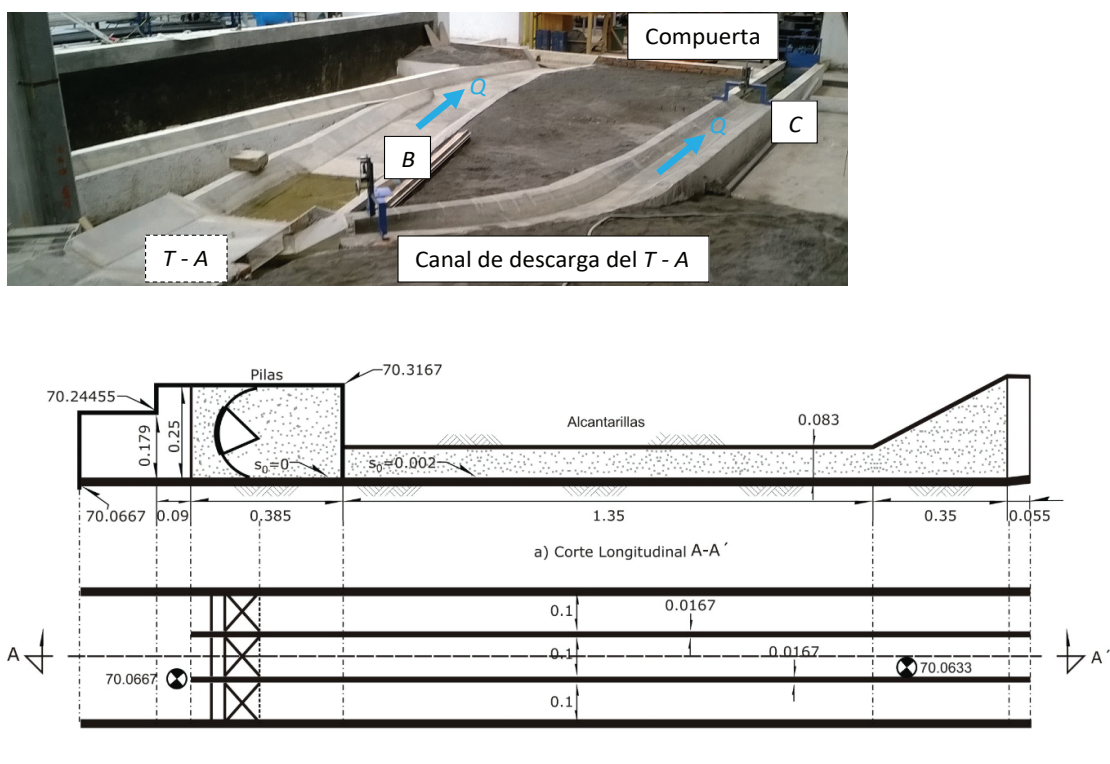

70.0667

b) Vista en planta

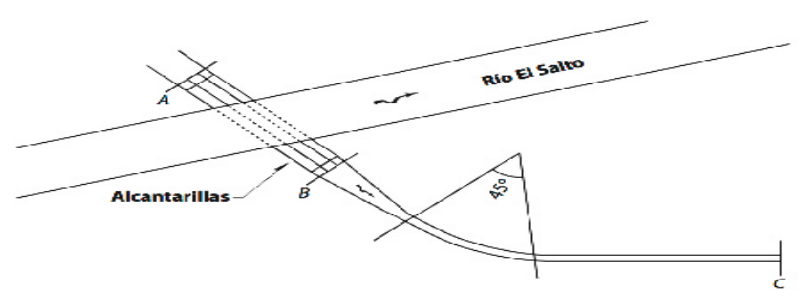

Figura 3. Canal con curvatura horizontal en el que descargan las alcantarillas

Figura 4a. Esquemas de la estructura de control y alcantarillas (acotaciones en $\mathrm{m}$ )

Figura 4b. Distribución de los limnímetros $A, B$ y $C$ en una parte del modelo físico
$0.083 \mathrm{~m}$. La pendiente de la plantilla de las alcantarillas es de 0.002 y su longitud es de 1.70 m (Figura 4a).

A la salida de las alcantarillas existe un pequeño escalón ascendente; en la margen izquierda la altura que asciende el fondo es de $0.017 \mathrm{~m}$, mientras que en la margen derecha el desnivel es de $0.012 \mathrm{~m}$ (ver fotografía del lado derecho en la Figura 2).

Para conectar la salida de las alcantarillas al canal en que descargan estas, se tiene una transición gradual recta de $1 \mathrm{~m}$ de longitud (Figura 5); en esta transición se cambia gradualmente de sección transversal rectangular a trapecial.

El ancho de plantilla del canal que sigue al terminar la transición gradual, varía entre 0.23 y 0.30 m; los taludes son del orden de 1.50: 1; este tramo de canal, de7.5m de longitud, inicia aguas abajo de donde termina la transición recta, con una curva horizontal de $45^{\circ}$ y pendiente de plantilla adversa (Figura 5).

\section{INFRAESTRUCTURA DE LA INSTALACIÓN EXPERIMENTAL}

El gasto de diseño del $T-A$, es de $0.0101 \mathrm{~m}^{3} / \mathrm{s}$. Para aforar el caudal en el modelo físico, se dispone de un vertedor de pared delgada y sección de forma triangular, cuyo ángulo central es de $90^{\circ}$. El cálculo del caudal que descarga el vertedor se obtiene con la fórmula recomendada en ISO 1438 (2017):

$Q \cong C \frac{8}{15} \tan 45^{\circ} \sqrt{2 g} h_{v}^{2.5}$

Donde:

$h_{v}=$ la carga en el vertedor triangular, en $m$

$Q$ = caudal que descarga dicho vertedor, en $\mathrm{m}^{3} / \mathrm{s}$

Respecto al valor empírico de C, en ISO 1438 (2017) se presenta una figura de donde se deduce que $C \cong 0.577$ (para venas liquidas totalmente aireadas); en el valle de México es común aceptar que $g \cong 9.79 \mathrm{~m} / \mathrm{s}^{2}$. Al sustituir los correspondientes valores en la ecuación (1), y después de hacer las operaciones se obtiene la expresión siguiente:

$Q \cong 1.361 h_{v}^{25}$ 


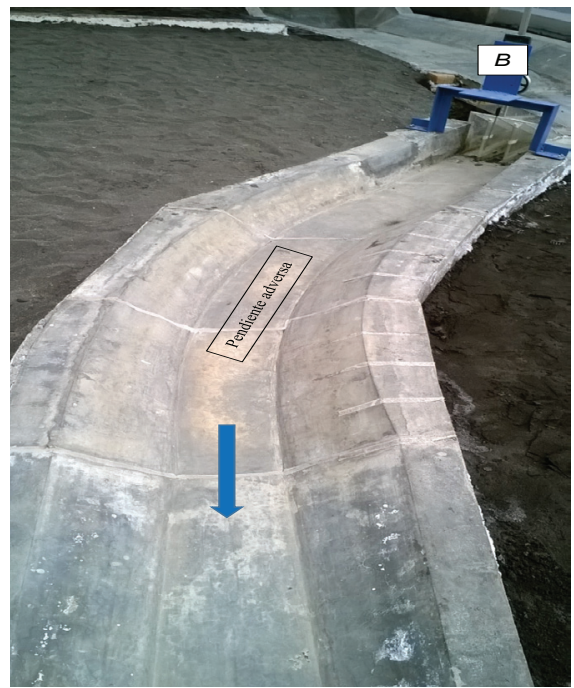

Figura 5. Tramo del canal con curvatura horizontal y pendiente adversa

La medición de los niveles del agua en el modelo se hizo con tres limnímetros: uno de ellos, denotado como $(A)$, se colocó aguas arriba de la entrada de las alcantarillas, a $0.10 \mathrm{~m}$ de la nariz de las pilas (Figura 2); otro (B), aguas abajo de las alcantarillas, a $0.20 \mathrm{~m}$ de donde terminan las pilas (Figura 2); y uno más $(C)$, en el extremo final del tramo que está aguas abajo de la curva horizontal (Figura 3).

\section{CONDICIÓN DE FRONTERA AGUAS ABAJO}

Aguas abajo del sistema de canales existe una obra de toma de una planta de tratamiento; se sabe que para tener un adecuado funcionamiento hidráulico de la planta de tratamiento, en la obra de toma el nivel de la superficie libre del agua debe tener la cota 70.1667 msnm, para cualquier caudal; por ello, en el modelo físi- co se colocó una compuerta plana vertical (Figura 3), cuya operación permite controlar el nivel de la superficie libre del agua. En cada uno de los experimentos se adecuó la apertura de la compuerta para tener siempre la mencionada cota del agua al final del canal trapecial. El tirante en esa sección transversal debe ser $y=0.0732 \mathrm{~m}$.

\section{LEY DE DESCARGA CON COMPUERTAS TOTALMENTE ABIERTAS}

Por un lado, en el extremo final del modelo físico la elevación de la superficie libre del agua debe tener la cota $\mathrm{H}=70.1667 \mathrm{~m}$; por otro lado, la elevación de la plantilla del canal en el extremo final de las alcantarillas es $70.0640 \mathrm{~m}$, y como la altura de las alcantarillas es de $0.0833 \mathrm{~m}$, la elevación de la clave de las mismas en la salida es de $70.1473 \mathrm{~m}$ (Figura 6); ahí se nota que el nivel de la superficie libre del agua, dado por la operación de la obra de toma, hace que tanto la salida de las alcantarillas, como la entrada de las mismas, sea ahogada.

\section{ESTUDIO EXPERIMENTAL}

Los caudales empleados en las pruebas experimentales desarrolladas en el modelo físico se reportan en la Tabla 1 ; para cada gasto se registraron las cotas del agua en las secciones $A, B$ y $C$. En cada prueba, se estableció flujo en régimen permanente. En la Tabla 1: $Q$, es el caudal calculado con la ecuación (2); $H_{A}$, la elevación de la superficie libre del agua en la ubicación del limnímetro $A$; $H_{B}$, la cota del agua en la localización del limnímetro $B$; y $H_{C}$, el nivel del agua en la ubicación del limnímetro $C$.

En la Figura 7 se incluyen los puntos asociados a las parejas de valores elevaciones - caudales medidos en las secciones $A$ y $B$, que corresponden a las secciones aguas arriba (puntos de color rojo) y aguas abajo (puntos de color azul) de las alcantarillas, respectivamente.

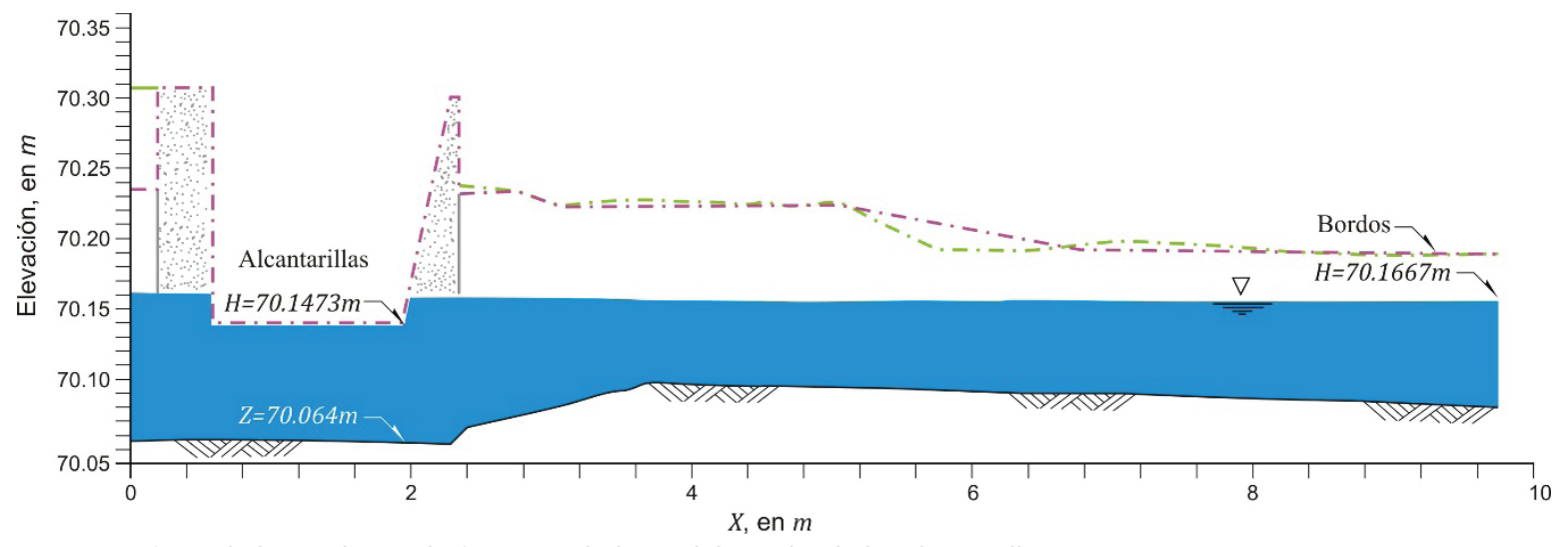

Figura 6. Efecto de la condición de frontera a lo largo del canal y de las alcantarillas 
Calibración de la ley de DESCARGa CON COMPUERTAS TOTALMENTE ABIERTAS

Los resultados experimentales (puntos), mostrados en la Figura 7, permiten determinar el coeficiente de rugosidad de Manning del modelo físico, y calibrar los coeficientes para el cálculo de las pérdidas locales de energía, debidas a los efectos de contracción y expansión ocasionadas por la entrada y la salida de las alcantarillas, respectivamente. El proceso de cálculo se reporta con mayor detalle en Hernández (2019), quien empleó el sistema de modelación numérica HEC - RAS (U.S. Army Corps of Engineering, 2010), para el cálculo de perfiles hidráulicos de flujo gradualmente variado, con flujo unidimensional y régimen permanente. Los coeficientes con los que se logra mejor aproximación a las mediciones de laboratorio son: coeficiente de rugosidad de Manning $n=0.010$, coeficiente de pérdida local

Tabla 1. Valores de caudales y cotas del agua registrados en las pruebas experimentales

\begin{tabular}{llll}
\hline$Q_{,}\left(\mathrm{m}^{3} / \mathrm{s}\right)$ & $H_{A^{\prime}}(\mathrm{m})$ & $H_{B^{\prime}}(\mathrm{m})$ & $H_{C^{\prime}}(\mathrm{m})$ \\
\hline 0.009594 & 70.1909 & 70.1802 & 70.1667 \\
0.009097 & 70.1880 & 70.1779 & 70.1647 \\
0.008261 & 70.1848 & 70.1755 & 70.1667 \\
0.008214 & 70.1860 & 70.1765 & 70.1667 \\
0.007900 & 70.1833 & 70.1746 & 70.1667 \\
0.007325 & 70.1812 & 70.1734 & 70.1675 \\
0.007032 & 70.1792 & 70.1725 & 70.1667 \\
0.006117 & 70.1771 & 70.1713 & 70.1664 \\
0.006091 & 70.1778 & 70.1716 & 70.1686 \\
0.006077 & 70.1767 & 70.1707 & 70.1667 \\
0.005714 & 70.1763 & 70.1708 & 70.1667 \\
0.004423 & 70.1728 & 70.1685 & 70.1667 \\
0.004390 & 70.1719 & 70.1683 & 70.1667 \\
0.004293 & 70.1728 & 70.1695 & 70.1667 \\
0.003144 & 70.1707 & 70.1674 & 70.1675 \\
0.001185 & 70.1675 & 70.1658 & 70.1667 \\
\hline
\end{tabular}

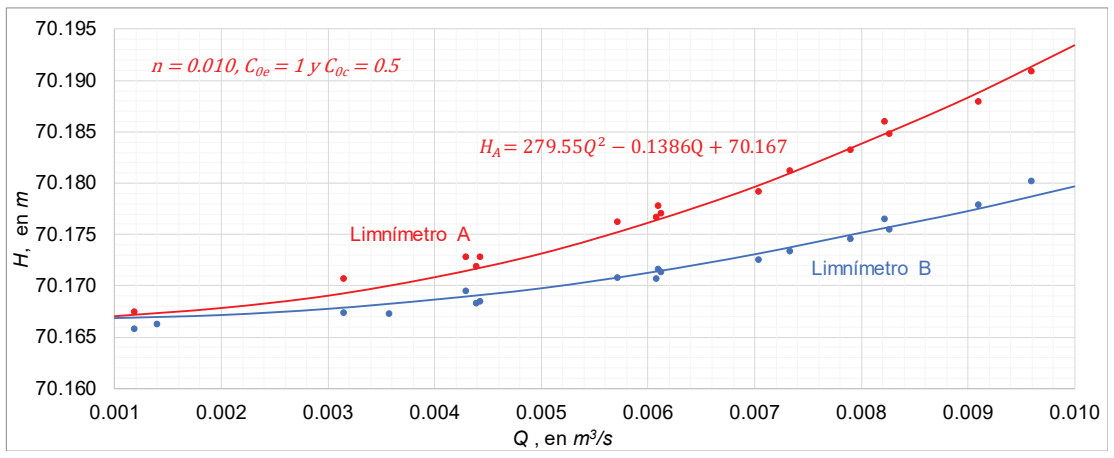

Figura 7. Curvas de descarga, aguas arriba y aguas abajo de las alcantarillas de energía por contracción $C_{0 c}=0.5$ (en la entrada de las alcantarillas), y el coeficiente de pérdida local de energía por expansión $C_{0 e}=1.0$ (en la salida de las alcantarillas). Los perfiles hidráulicos calculados se presentan en la Figura 8, y corresponden a los de mejor ajuste de los coeficientes mencionados (Hernández, 2019).

Por un lado, en la Figura 7, los puntos rojos corresponden a las elevaciones de la superficie libre del agua registradas con el limnímetro $A$, mientras que los puntos de color azul están asociados al empleo y localización del limnímetro $B$. Por otro lado, se tienen las coordenadas elevaciones - gastos que fueron obtenidas con el cálculo de perfiles hidráulicos, en las mismas secciones transversales en las que se localizan los limnímetros $A$ y $B$; estas últimas coordenadas se utilizan para definir las curvas con líneas en color rojo y azul que también están incluidas en la Figura 7, respectivamente. 


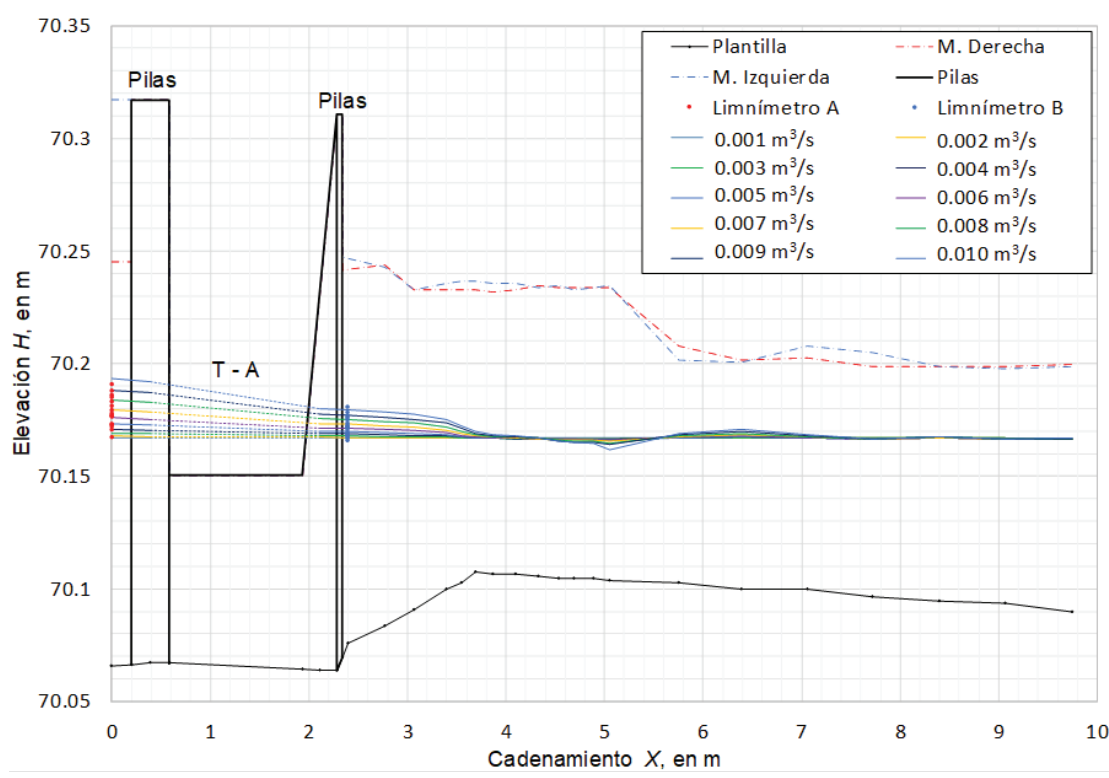

Figura 8. Perfiles hidráulicos calculados con el sistema de modelación HEC - RAS
El modelo numérico de la ley de descarga que corresponde al tramo que está aguas arriba de las alcantarillas, cuando las compuertas de la estructura de control están totalmente abiertas, es el que mejor representa a la curva en color rojo de la Figura 7. Una manera sencilla de obtener la ecuación de mejor ajuste se basa en emplear una de las herramientas de cálculo del paquete Excel; así se obtuvo la expresión siguiente

$H_{A}=279.55 Q^{2}-0.1386 Q+70.167$

La ecuación (3) se emplea como condición de frontera para calcular el caudal que descargan las compuertas radiales parcialmente abiertas, cuya descarga es ahogada.

\section{LEY DE DESCARGA CON COMPUERTAS PARCIALMENTE ABIERTAS}

El notable efecto de remanso hacia aguas arriba de las alcantarillas se propicia por los factores siguientes: la condición de frontera que fija la elevación de la superficie libre del agua en la ubicación del limnímetro $C$, la pendiente adversa que se tiene en el tramo que está aguas abajo de las alcantarillas, y la presencia de las alcantarillas. Esto ocasiona que al abrir parcialmente las compuertas radiales que están aguas arriba de las alcantarillas, la descarga se ahogue.

Dos de los estudios más recientes respecto al funcionamiento hidráulico de compuertas radiales, con descarga sumergida, son los publicados por Bijankhan et al. (2013) y Abdelhaleem (2016). Bijankhan et al. (2013) emplean las contribuciones presentadas por Ferro (2000), Shahrokhnia y Javan (2006), y los extensos estu- dios y resultados experimentales de Buyalski (1983); Abdelhaleem (2016) agrega un parámetro y usa gran cantidad de mediciones realizadas en campo.

Formulación de BijanKHan et AL. (2013)

Ferro (2000), con base en el análisis dimensional, propone una expresión para calcular el caudal que descarga una compuerta vertical plana; después, Shahrokhnia y Javan (2006) extienden el citado estudio a compuertas radiales; posteriormente, Bijankhan et al. (2013) emplean el mismo enfoque y utilizan los extensos resultados experimentales de Buyalski (1983), para proponer varias metodologías para calcular la ley de descarga de compuertas radiales, en condiciones de descarga libre y sumergida.

Los experimentos reportados por Buyalski (1983) incluyen condiciones tanto para compuertas con descarga libre como sumergida, y se realizaron en un canal de sección rectangular, con ancho de plantilla $B=0.762 \mathrm{~m}$, con una compuerta radial de ancho $b=0.711 \mathrm{~m}$ y radio $R=0.702 \mathrm{~m}$; la diferencia del ancho $B$ del canal respecto al ancho $b$ de la compuerta, se debe a que en el lado izquierdo del canal se colocó la mitad de una pila. Se utilizaron tres alturas, $h$, del perno de la compuerta, 0.409, 0.461 y $0.511 \mathrm{~m}$; también se emplearon dos tipos de sellos que se colocan comúnmente en el labio inferior de la compuerta (Figura 9): uno de ellos consiste en una goma dura y el otro se distingue por tener forma de nota musical; Asimismo, el autor realizó pruebas con el borde agudo (sin sello); y trabajó con nueve combinaciones de montaje, pues para cada una de las condicio- 


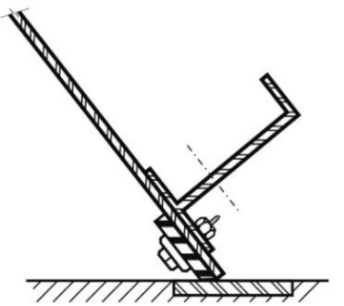

Barra de goma dura

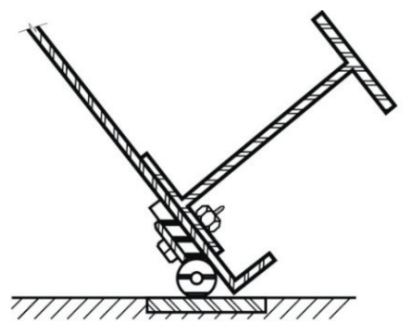

Nota músical

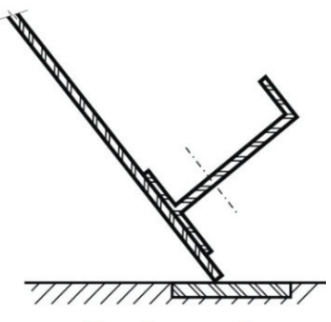

Borde agudo
Figura 9. Tipos de sello empleados por Buyalski (1983)

nes del labio inferior se utilizaron las tres alturas de perno citadas (Tabla 2).

Tabla 2. Características de las pruebas experimentales de Buyalski (1983)

\begin{tabular}{ccc}
\hline Prueba & Tipo de sello & $h,(m)$ \\
\hline$G-1$ & Goma dura & 0.461 \\
G -2 & Goma dura & 0.511 \\
G -3 & Goma dura & 0.409 \\
$G-4$ & Nota musical & 0.409 \\
$G-5$ & Nota musical & 0.461 \\
$G-6$ & Nota musical & 0.511 \\
$G-7$ & Borde agudo & 0.409 \\
$G-8$ & Borde agudo & 0.461 \\
$G-9$ & Borde agudo & 0.511 \\
\hline
\end{tabular}

Las expresiones propuestas por Bijankhan et al. (2013) para calcular el caudal que descarga una compuerta radial, con desfogue sumergido, son las siguientes; primero, es necesario calcular el ángulo $\theta$ (en radianes), formado entre la horizontal y la tangente al labio inferior de la compuerta (Figura 10); este se obtiene con base en la geometría de las compuertas, con la expresión siguiente:

$\theta=\operatorname{angcos}\left[\frac{(h-w)}{R}\right]$

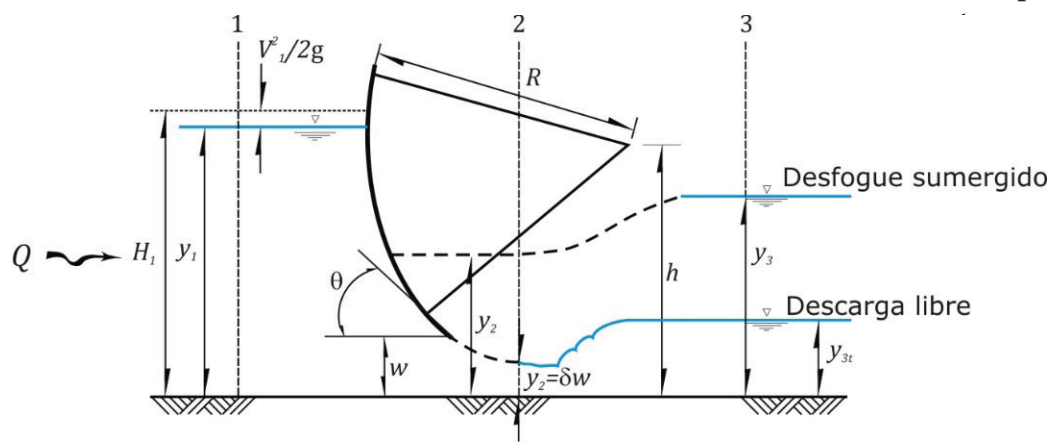

Figura 10. Características de la descarga libre y sumergida de una compuerta radial 
Tabla 3. Parámetros de la ecuación (6), (Bijankhan et al., 2013)

\begin{tabular}{cccccc}
\hline Prueba & $a_{0}$ & $b_{1}$ & $\alpha$ & $\beta$ & $b_{2}$ \\
\hline G - 1 & 0.8100 & 0.4340 & 3.0569 & 0.8171 & 0.2958 \\
G - 2 & 0.8069 & 0.4160 & 2.3252 & 0.8237 & 0.3092 \\
G - 3 & 0.7936 & 0.4200 & 2.8600 & 0.8163 & 0.2949 \\
G - 4 & 0.7793 & 0.4186 & 5.8204 & 0.8177 & 0.2097 \\
G - 5 & 0.7804 & 0.4220 & 7.9079 & 0.8168 & 0.1804 \\
G - 6 & 0.7904 & 0.4265 & 9.9990 & 0.8155 & 0.1691 \\
G - 7 & 0.7838 & 0.4212 & 5.2643 & 0.8151 & 0.2204 \\
G - 8 & 0.7851 & 0.4277 & 5.1240 & 0.8070 & 0.2211 \\
G - 9 & 0.7860 & 0.4371 & 6.1956 & 0.8350 & 0.2043 \\
\hline
\end{tabular}

(Compuerta radial con $R=0.702 \mathrm{~m}$ y $b=0.711 \mathrm{~m})$.
Bijankhan et al. (2013) definen la llamada función de Reducción de caudal, $\phi\left(\prod\right)$, de la ecuación (6), si la descarga es sumergida:

$$
\phi(\Pi)=\left[\frac{\frac{\left(y_{1}-y_{3}\right)}{w}}{\alpha\left[\frac{\left(y_{3}-y_{3 t}\right)}{w}\right]^{\beta}+\frac{\left(y_{1}-y_{3}\right)}{w}}\right]^{b^{2}}
$$

Los valores de los parámetros $\alpha, \beta, \mathrm{y} b_{2}$, de la ecuación (8) se reportan en la Tabla 3.

En el caso de que la compuerta funcione hidráulicamente con descarga libre, y se presente un salto hidráulico claro, el tirante conjugado menor es el de la vena contracta $\left(y_{2}=\delta w\right.$, Figura 10); el correspondiente tirante conjugado mayor, $y_{3 \text { t, }}$ permite establecer un límite, que al ser excedido da lugar a que la descarga de la compuerta se ahogue. Lin et al. (2002) proponen calcular el tirante $y_{3 t}$ con la expresión siguiente:

$\frac{y_{3 t}}{\delta w}=\frac{1}{2}\left[\sqrt{1+\frac{16}{\frac{\delta w}{y_{1}}\left(\frac{\delta w}{y_{1}}+1\right)}}-1\right]$

El tirante $y_{3 t}$ se compara con el tirante $y_{3}$, que es el que se obtiene del cálculo del remanso producido por las alcantarillas. En el caso de que las compuertas radiales estén parcialmente abiertas, el tirante $y_{3}$ cambia únicamente en función del caudal; la expresión que relaciona el caudal con el nivel de la superficie libre del agua $\left(H_{A}\right)$, en la entrada de las alcantarillas, y hace que las compuertas radiales funcionen con descarga ahogada, se obtiene con la ecuación (3); por ello, para calcular el tirante $y_{3}$, primero se emplea la ecuación (3), para calcular $H_{A}$ y al resultado se le resta la elevación de la plantilla del canal.

La ecuación (8) es válida cuando se cumple que $y_{3}>$ $y_{3 t}$ (Figura 10); en el caso particular de que $y_{3}=y_{3 \prime}$ entonces la función de reducción de caudal, ecuación (8), es igual a la unidad, y se tienen condiciones de descarga libre de la compuerta (salto hidráulico claro). En Jiménez (2017) se presenta un estudio en el que se emplea el mismo tipo de fórmula adimensional para el caso de compuertas radiales con descarga libre. En el caso de que la compuerta esté parcialmente abierta y la descarga sea libre, el tirante $y_{3}$ sería menor que $y_{3 t}$ y ya no sería válida la ecuación (8).

\section{VALORES DE LOS PARÁMETROS}

Las compuertas que se estudian en este trabajo no tienen ningún sello en el labio inferior; por ello, los parámetros a emplear deben ser los obtenidos de las series de pruebas $G-7, G-8$ y $G-9$, cuyos valores se reportan en la Tabla 3. Estos parámetros fueron calibrados y verificardos por Bijankhan et al. (2013), con base en las mediciones de Buyalski (1983). Existe la incertidumbre respecto a la validez de estos parámetros para otras dimensiones de las compuertas.

Al observar la Tabla 3, con excepción de $\alpha$, los valores son aproximadamente constantes para cada parámetro; por ello, se decidió emplear los valores medios de los parámetros, los cuales son: $\bar{a}_{0}=0.785, \bar{b}_{1}=0.429$, $\bar{b}_{2}=0.2453, \bar{\beta}=0.819$ y $\bar{\alpha}=5.528$.

\section{Metodología de CálCulo}

$\mathrm{Al}$ inspeccionar el modelo numérico propuesto por Bijankhan et al. (2013), se nota que una manera de resolver las ecuaciones (6), (7) y (8), consiste en emplear el 
método numérico conocido como de punto fijo, (Burden y Faires, 2011), es decir, se requiere emplear un proceso iterativo.

A continuación se propone una metodología que incluye la aplicación del método numérico. Los datos son: altura del perno de la compuerta, $h=0.105 \mathrm{~m}$; radio de la compuerta, $R=0.12 \mathrm{~m}$; ancho de cada compuerta, $b=0.10 \mathrm{~m}$; aceleración de la gravedad, $g=9.79 \mathrm{~m} / \mathrm{s}^{2}$ (para la Ciudad de México). Los cálculos se desarrollan para dos aberturas de las compuertas, $w=\{0.016 \mathrm{~m}$, $0.033 \mathrm{~m}\}$. Las variables a calcular son: el caudal $Q$ que descargan las compuertas, en $\mathrm{m}^{3} / \mathrm{s}$; el tirante $y_{1}$ aguas arriba de las compuertas, en $\mathrm{m}$; $\mathrm{y}$ el tirante $y_{3}$ aguas abajo de las compuertas, con el que se ahoga la descarga. La metodología es la siguiente:

a) Se propone la abertura $w$ de la compuerta

b) Se calcula el ángulo $\theta$

$$
\theta=\operatorname{ang} \cos \left[\frac{(h-w)}{R}\right]
$$

c) Se calcula el coeficiente de contracción $\delta$

$$
\delta=1.001-0.2349 \theta-0.1843 \theta^{2}+0.1133 \theta^{3}
$$

d) Se propone un valor inicial del tirante $y_{1}$, aguas arriba de las compuertas

e) Se calcula el tirante máximo, $y_{3 t}$ que delimita las condiciones de descarga libre de la compuerta

$$
y_{3 t}=\frac{\delta w}{2}\left[\sqrt{1+\frac{16}{\frac{\delta w}{y_{1}}\left(\frac{\delta w}{y_{1}}+1\right)}}-1\right]
$$

f) Se propone un valor del caudal $Q$

g) Se calcula el tirante $y_{3}\left(y_{3}=H_{A}-Z_{A}\right)$, que se localiza aguas arriba de las alcantarillas; para ello se emplea la ecuación (3):

$$
y_{3}=H_{A}-70.067=279.55 Q^{2}-0.1386 Q+0.10
$$

h) Se comparan los valores de los tirantes $y_{3}$ y $y_{3 i}$; cuando $y_{3}>y_{3 t}$, la descarga de las compuertas es ahogada

i) Se calcula la función de reducción de caudal

$$
\phi(\Pi)=\left[\frac{\frac{\left(y_{1}-y_{3}\right)}{w}}{\alpha\left[\frac{\left(y_{3}-y_{3 t}\right)}{w}\right]^{\beta}+\frac{\left(y_{1}-y_{3}\right)}{w}}\right]^{b_{2}}
$$

j) Se calcula la variable $K$

$$
\frac{K}{w}=a_{0}\left(\frac{y_{1}}{w}\right)^{b_{1}} \phi(\Pi)
$$

k) Se calcula el caudal $Q$ $Q=\sqrt{g} b K^{3 / 2}$

1) Se compara el caudal calculado en el paso k) con el propuesto en el paso f); si no son iguales, se repite el cálculo a partir del paso f), con el valor del caudal ob-

Tabla 4. Iteraciones para obtener el caudal con $w=0.016 \mathrm{~m}$ y $y_{1}=0.15 \mathrm{~m}$

\begin{tabular}{ccccc}
\hline$Q,\left(m^{3} / \mathrm{s}\right)$ & $y_{3}(m)$ & $\phi(\Pi)$ & $K,(m)$ & $Q_{,}\left(\mathrm{m}^{3} / \mathrm{s}\right)$ \\
\hline 0.00320587 & 0.10269974 & 0.75157116 & 0.02465673 & 0.00363426 \\
0.00363426 & 0.10343759 & 0.74695061 & 0.02450514 & 0.00360080 \\
0.00360080 & 0.10337638 & 0.74733284 & 0.02451768 & 0.00360356 \\
0.00360356 & 0.10338141 & 0.74730141 & 0.02451665 & 0.00360333 \\
0.00360333 & 0.10338100 & 0.74730399 & 0.02451673 & 0.00360335 \\
0.00360335 & 0.10338103 & 0.74730378 & 0.02451673 & 0.00360335 \\
\hline
\end{tabular}

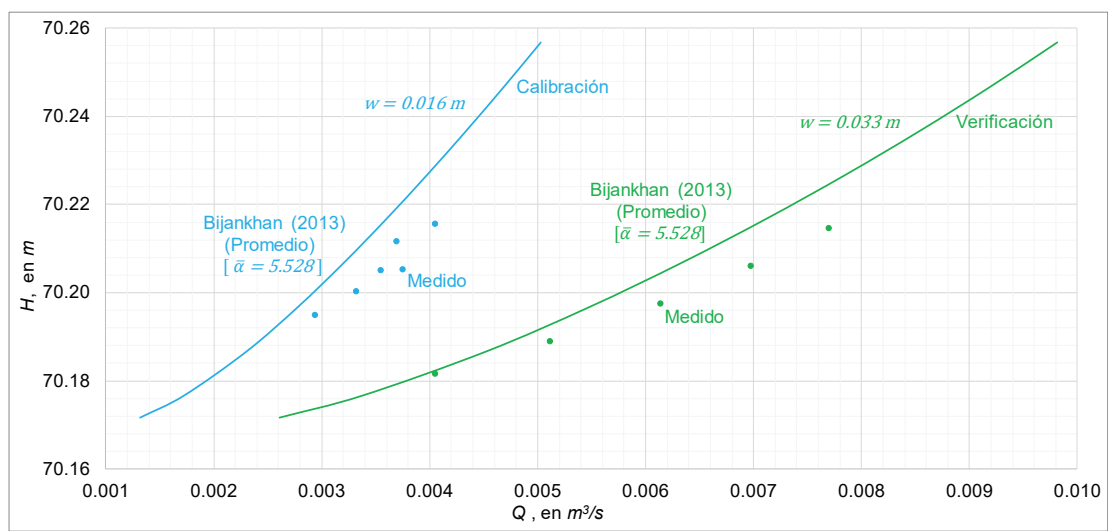

Figura 11. Leyes de descarga calculadas con los parámetros $\bar{a}_{0}, \bar{b}_{1}, \bar{b}_{2}, \bar{\beta}$ y $\bar{\alpha}$ 
tenido en el paso k), hasta que los gastos sean aproximadamente iguales (con una tolerancia prefijada).

En la Tabla 4 se presentan las iteraciones para la abertura $w=0.016 \mathrm{~m}$ con el tirante $y_{1}=0.15 \mathrm{~m}$. En este caso, con 6 iteraciones, los caudales son iguales con ocho cifras significativas. En la tercera iteración, el error absoluto es menor que $0.1 \%$.

En la Figura 11 se presentan las curvas elevaciones caudales calculadas con las dos aberturas propuestas de las compuertas y los valores medidos en el laboratorio.

\section{CALIBRACIÓN Y VERIFICACIÓN DEL MODELO NUMÉRICO}

Al comparar los valores que forman las curvas de descarga calculadas con el modelo numérico, con las mediciones obtenidas en laboratorio, se nota que para la misma elevación, el caudal calculado es menor que el medido (del orden de $-11 \%$ ), para ambas aberturas de las compuertas; esto indica la necesidad de calibrar y verificar el modelo numérico de Bijankhan et al. (2013). Para ello, se propone modificar el valor del parámetro $\alpha$, pues este es el que más varía. Así, se realizó un análisis de sensibilidad proponiendo distintos valores para el parámetro $\alpha=\{4,3.5,3\}$, los resultados indican que para una misma elevación, el caudal aumenta conforme el valor de $\alpha$ disminuye. Por prueba y error se llegó a que con el valor $\alpha=3.3$, se logra una adecuada concordancia con las mediciones (Figura 12); en esta misma figura se incluye la curva del funcionamiento con las compuertas abiertas y los valores medidos. En la Tabla 5 se reportan los resultados obtenidos del modelo numérico con ambas aberturas.

\section{ANÁLISIS DE CONFIABILIDAD}

Se calcularon los errores relativos con la expresión siguiente:
Error $=\left(\frac{Q_{\text {calculado }}-Q_{\text {medido }}}{Q_{\text {medido }}}\right) x 100$

En las Tablas 6a y 6b se presentan los errores calculados. Se observa que el error obtenido para las pruebas no supera $5 \%$, con excepción del caudal $Q=0.00405$ $\mathrm{m}^{3} / \mathrm{s}$, con $w=0.033 \mathrm{~m}$, que es de $8.4 \%$.

También se calculó el porcentaje de error absoluto medio, PEAM, con la expresión siguiente:

$\operatorname{PEAM} \frac{\sum\left|\frac{Q_{\text {calculado }}-Q_{\text {medido }}}{Q_{\text {medido }}} \times 100\right|}{N}$

donde $N$ es el número de datos para cada apertura; para $w=0.016 \mathrm{~m}$, se tiene un PEAM de $2.59 \%$ y para $w=0.033 \mathrm{~m}$, el PEAM es de $2.92 \%$; esto permite concluir que la aproximación del modelo numérico calibrado y verificado, tiene una aproximación mayor que $95 \%$.

\section{Formulación de AbDelhaleEm (2016)}

Esta formulación es similar a la propuesta por Bijankhan et al. (2013); la principal diferencia consiste en que se incluye un parámetro adimensional, el cual relaciona el ancho $B$ del canal de llegada al tramo de pilas y compuertas, con el ancho total $b$ de todas las compuertas colocadas en paralelo. El modelo propuesto es el siguiente:

$\frac{Q}{b w^{1.5} \sqrt{g}}=a_{3}\left(\frac{y_{1}}{w}\right)^{b_{3}}\left(\frac{b}{B}\right)^{c_{3}}\left[\frac{\left(y_{1}-y_{3}\right)}{\lambda\left(y_{1}-y_{3 t}\right)+\left(y_{1}-y_{3}\right)}\right]^{d_{3}}$

En este modelo se requieren ajustar cinco parámetros. El autor señala que los valores de los parámetros $a_{3}, b_{3}$, $c_{3}, d_{3}$ y $\lambda$ deben obtenerse con base en mediciones de

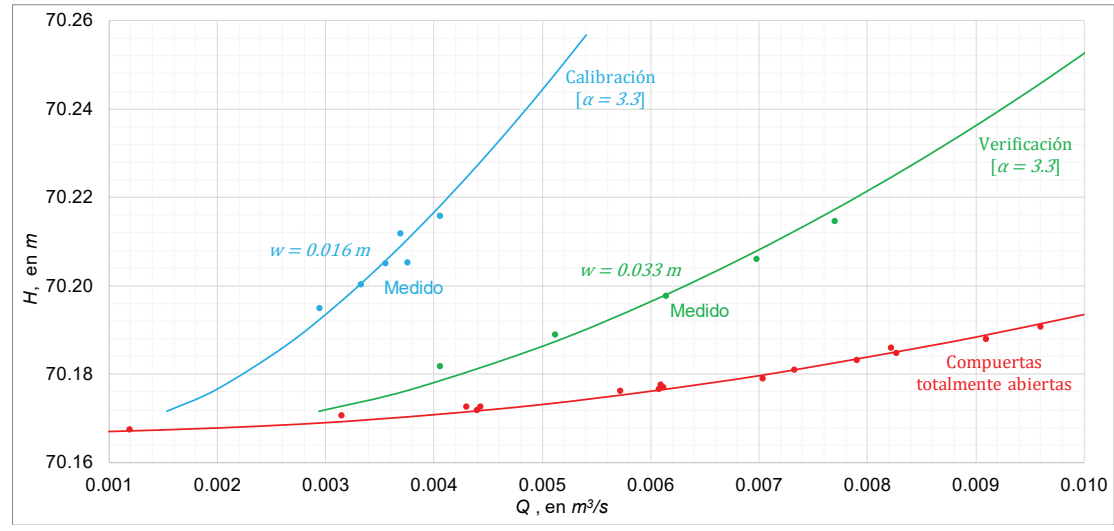

Figura 12. Leyes de descarga calculadas con los parámetros $\bar{a}_{0}, \bar{b}_{1}, \bar{b}_{2}, \bar{\beta}$ y $\alpha=3.3$ 
Tabla 5. Resultados del modelo numérico con $\bar{a}_{0}, \bar{b}_{1}, \bar{b}_{2}, \bar{\beta}$ y $\bar{\alpha}=3.3$

\begin{tabular}{cccc}
\hline & & $w=0.016 \mathrm{~m}$ & $w=0.033 \mathrm{~m}$ \\
\hline$y_{,}(m)$ & $H_{,}(m)$ & \multicolumn{2}{c}{$Q_{,}\left(\mathrm{m}^{3} / \mathrm{s}\right)$} \\
\hline 0.105 & 70.1717 & 0.001535394 & 0.00293545 \\
0.11 & 70.1767 & 0.001999510 & 0.00379569 \\
0.12 & 70.1867 & 0.002643214 & 0.00503310 \\
0.13 & 70.1967 & 0.003151599 & 0.00601823 \\
0.14 & 70.2067 & 0.003596873 & 0.00687656 \\
0.15 & 70.2167 & 0.004003355 & 0.00765191 \\
0.16 & 70.2267 & 0.004382392 & 0.00836543 \\
0.17 & 70.2367 & 0.004740303 & 0.00902948 \\
0.18 & 70.2467 & 0.005081041 & 0.00965230 \\
0.19 & 70.2567 & 0.005407300 & 0.01023983 \\
\hline
\end{tabular}

Tabla 6a. Comparación entre los caudales medidos y calculados, con $w=0.016 \mathrm{~m}$

\begin{tabular}{cccc}
\hline \multicolumn{4}{c}{$Q,\left(\mathrm{~m}^{3} / \mathrm{s}\right)$} \\
\hline$H_{A^{\prime}}(\mathrm{m})$ & Medido & Calculado & Error \\
\hline 70.19496 & 0.00294 & 0.00307127 & $4.5 \%$ \\
70.2004 & 0.00332 & 0.00334022 & $0.6 \%$ \\
70.2051 & 0.003548 & 0.00355403 & $0.2 \%$ \\
70.2118 & 0.00369 & 0.00383379 & $3.9 \%$ \\
70.2054 & 0.00375 & 0.00356715 & $-4.9 \%$ \\
70.2158 & 0.00405 & 0.0039892 & $-1.5 \%$ \\
\hline
\end{tabular}

Tabla 6b. Comparación entre los caudales medidos y calculados, con $w=0.033 \mathrm{~m}$

\begin{tabular}{cccc}
\hline \multicolumn{4}{c}{$Q,\left(\mathrm{~m}^{3} / \mathrm{s}\right)$} \\
\hline$H_{A^{\prime}}(\mathrm{m})$ & Medido & Calculado & Error \\
70.1818 & 0.00405 & 0.00439165 & $8.4 \%$ \\
70.189 & 0.005113 & 0.00523745 & $2.4 \%$ \\
70.1977 & 0.00614 & 0.00612718 & $-0.2 \%$ \\
70.2061 & 0.006973 & 0.00687138 & $-1.5 \%$ \\
70.2147 & 0.0077 & 0.00754064 & $-2.1 \%$ \\
\hline
\end{tabular}

Tabla 7. Parámetros de la ecuación (10), obtenidos por Abdelhaleem (2016)

\begin{tabular}{cccccc}
\hline Estructura & $a_{3}$ & $b_{3}$ & $c_{3}$ & $d_{3}$ & $\lambda$ \\
\hline Al-Tawfiki & 0.816 & 0.217 & 1.153 & 0.551 & 0.784 \\
Al-Menoufi & 0.291 & 0.579 & 4.387 & 0.182 & 0.628 \\
Abasi & 0.873 & 0.270 & 1.046 & 0.391 & 1.335 \\
\hline
\end{tabular}




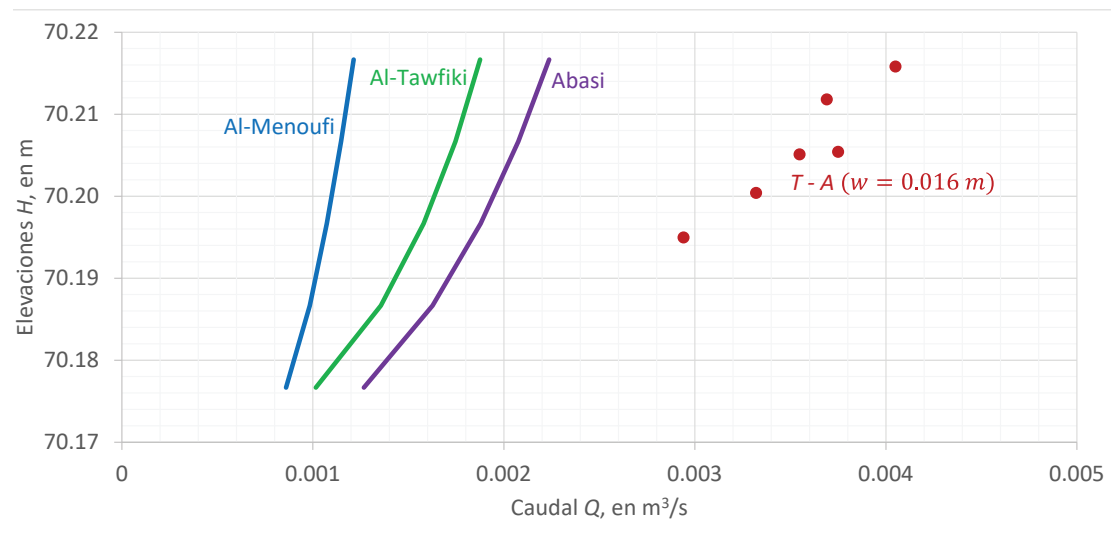

Figura 13. Comparación del modelo numérico (10) con mediciones en el $T$ - A laboratorio o de campo para cada caso particular, lo cual es una desventaja. Así, Abdelhaleem (2016) hizo gran cantidad de mediciones de campo en tres estructuras de control; con esta información calibró los parámetros de la ecuación (10); en la Tabla 7 se reportan los correspondientes valores para cada una de las tres estructuras de control.

Desafortunadamente, los valores de los parámetros obtenidos por Abdelhaleem (2016) no presentan ninguna tendencia; sin embargo, se propuso emplear el modelo numérico en cuestión, (ecuación (10)), con cada serie de valores reportados en la Tabla 7. En la Figura 13 se incluyen las curvas de descarga calculadas con la abertura $w=0.016 \mathrm{~m}$ y también los valores medidos en las pruebas experimentales del $T-A$.

Las diferencias entre los valores calculados y medidos son mayores que $50 \%$; por ello, se consideró que no es factible desarrollar el proceso de calibración y verificación del modelo numérico propuesto por Abdelhaleem (2016), en el presente trabajo.

\section{CONCLUSIONES Y RECOMENDACIONES}

Se dispone de un modelo numérico, propuesto por Bijankhan et al. (2013), para calcular las leyes de descarga de una estructura de control, en la que se tienen tres compuertas radiales que funcionan con descarga ahogada, por la presencia de tres alcantarillas. Las curvas de descarga se calibraron y verificaron con base en mediciones experimentales obtenidas en un modelo físico de la estructura de control. Así, se logró tener un modelo numérico con $95 \%$ de confiabilidad.

El modelo numérico propuesto por Bijankhan et al. (2013), del tipo adimensional, es relativamente nuevo; la presentación y aplicación de este método permite tener una alternativa confiable para calcular el caudal que descarga una compuerta radial, o un conjunto de compuertas radiales en paralelo, con desfogue sumergido.
Los resultados obtenidos con el modelo numérico propuesto por Abdelhaleem (2016), difieren notablemente respecto a los valores medidos, y por ello, se consideró que no era adecuado calibrar este modelo numérico para el caso tratado en este trabajo.

Los estudios presentados en Jiménez y Berezowsky (2009), Jiménez (2017) y el que se describe en este trabajo, demuestran que es indispensable calibrar y verificar los modelos numéricos que han sido propuestos para calcular el caudal que descargan las compuertas radiales; esto se debe a la complejidad de las características del flujo en la vecindad de las compuertas, tanto para la descarga libre como la sumergida. Aunque se tiene un buen avance en el cálculo de las leyes de descarga de compuertas radiales, el problema no está totalmente resuelto; esto da lugar a continuar haciendo estudios. Una alternativa de estudio consiste en emplear sistemas de modelación numérica para flujo tridimensional, y revisar que el sistema de modelación represente en forma adecuada efectos como la formación de remolinos en el flujo cercano a las compuertas, succión de aire, corrientes secundarias en el flujo que llega a la estructura de control, etcétera.

El modelo propuesto por Bijankhan et al. (2013), tiene como base la extensa cantidad de pruebas de laboratorio realizadas y publicadas por Buyalski (1983), quien utilizó dos tipos de sello en el labio inferior de las compuertas; desafortunadamente, en las pruebas experimentales los sellos se colocaron en el lado de la placa de aguas arriba, lo cual es contrario a la recomendación que consiste en colocar el sello del labio inferior de la compuerta en la parte de atrás de la placa, para reducir las vibraciones en la estructura. Esta diferencia permite indicar que solamente se utilicen los resultados obtenidos por Buyalski (1983), que corresponde a la condición en la que no se coloca ningún tipo de sello en el labio inferior de la compuerta. 


\section{Agradecimientos}

Agradecemos al M. en I. Víctor Manuel Ortiz-Martínez, junto con su grupo de trabajo, por colaborar en la preparación del modelo físico y en una parte del estudio experimental.

Asimismo, a las Licenciadas Josefina Elizabeth Plata-García y María Guillermina Sánchez-Nahuacatl de la Unidad de Servicios de Información y del Instituto de Ingeniería, UNAM, respectivamente, por el apoyo fundamental para conseguir una parte del material que se incluyó en las referencias, cuya consulta permitió enriquecer este trabajo académico.

A Israel García Castro, de la Sección de Dibujo del Instituto de Ingeniería, quien elaboró los esquemas que se incluyen en las figuras del presente trabajo.

\section{RefERENCIAS}

Abdelhaleem, F.S.F. (2016). Discharge estimation for submerged parallel radial gates. Flow Measurement and Instrumentation Journal, 52, Elsevier, 240-245. https://doi.org/10.1016/j.flowmeasinst.2016.11.001.

Bijankhan, M., Ferro, V. \& Kouchakzadeh, S. (2013). New stagedischarge relationships for radial gates. Journal of Irrigation and Drainage Engineering, ASCE, 139(5). http://doi.org/10.1061/ (ASCE)IR.1943-4774.0000556

Burden, R.L. \& Faires, J.D. (2011), Numerical Analysis, 9ª edición, EUA: Broks/Cale.

Buyalski, C.P. (1983). Discharge algorithms for canal radial gates, REC-ERC-83-9, Engineering and Research Center. U.S. Bureau of Reclamation, Denver.

Clemmens, A.J., Strelkoff, T.S. \& Replogle, J.A. (2003) Calibration of Submerged Radial Gates. Journal of Hydraulic Engineering, ASCE, 12(9). https://doi.org/10.1061/(ASCE)0733-9429(2003)129: 9(680)
Cozzo, G. (1978). Una formula per il calcolo del coefficiente d'efflusso delle luci sotto paratoie. L'Energia Elettrica, 11(12), 504-513.

Ferro, V. (2000). Simultaneous flow over and under gate. Journal of Irrigation and Drainage Engineering, ASCE, 126(3). http://doi. org/10.1061/(ASCE)0733-9437(2000)126:3(190)

Henderson, F.M. (1966). Open Channel Flow, New York: Mac Millan.

Hernández, L.E. (2019) Estudio para obtener las leyes de descarga de compuertas radiales con desfogue sumergido. Tesis de Licenciatura, Facultad de Ingeniería, Universidad Nacional Autónoma de México, México.

ISO 1438 (2017), Hydrometry-Open channel flow measurement using thin-plate weirs.

Jiménez, A.A. (2017) Calibración y verificación de las leyes de descarga de una estructura de control, México: División de Ingenierías Civil y Geomática, Facultad de Ingeniería, Universidad Nacional Autónoma de México.

Jiménez, A.A. \& Berezowsky, M. (2009) Modelación matemática de la operación de la estructura de control del río Carrizal, Serie Investigación y Desarrollo SID/662, México: Instituto de Ingeniería, Universidad Nacional Autónoma de México.

Lin, C.H., Yen, J.F. \&Tsai, C.T. (2002) Influence of sluice gate contraction coefficient on distinguishing condition. ASCE, Journal of Irrigation and Drainage Engineering, 128 (4). http://doi. org/10.1061/(ASCE)0733-9437(2002)128:4(249)

Shahroknia, M. \& Javan, M. (2006). Dimensioless stage-discharge relationship in radial gates. Journal of Irrigation and Drainage Engineering, ASCE, 132(2). http://doi.org/10.1061/(ASCE)07339437(2006)132:2(180)

Sotelo, A.J. (2002), Hidráulica General, Vol. 1, Fundamentos, México: Limusa.

Tel, J. (2000) Discharge relations for radial gates. MSc thesis, Delft, Technical University, Delft, The Netherlands.

U.S. ARMY CORPS OF ENGINEERS. HEC-RAS. (2010) Hydraulic reference manual and User's Manual Version 4. Davis, CA: U.S. Army Corps of Engineers. Hydrologic Engineer Center. 